\title{
VTCdb: a gene co-expression database for the crop species Vitis vinifera (grapevine)
}

\author{
Darren CJ Wong ${ }^{1}$, Crystal Sweetmann ${ }^{1}$, Damian P Drew ${ }^{1,2}$ and Christopher M Ford ${ }^{1 *}$
}

\begin{abstract}
Background: Gene expression datasets in model plants such as Arabidopsis have contributed to our understanding of gene function and how a single underlying biological process can be governed by a diverse network of genes. The accumulation of publicly available microarray data encompassing a wide range of biological and environmental conditions has enabled the development of additional capabilities including gene co-expression analysis (GCA). GCA is based on the understanding that genes encoding proteins involved in similar and/or related biological processes may exhibit comparable expression patterns over a range of experimental conditions, developmental stages and tissues. We present an open access database for the investigation of gene co-expression networks within the cultivated grapevine, Vitis vinifera.
\end{abstract}

Description: The new gene co-expression database, VTCdb (http://vtcdb.adelaide.edu.au/Home.aspx), offers an online platform for transcriptional regulatory inference in the cultivated grapevine. Using condition-independent and condition-dependent approaches, grapevine co-expression networks were constructed using the latest publicly available microarray datasets from diverse experimental series, utilising the Affymetrix Vitis vinifera GeneChip (16 K) and the NimbleGen Grape Whole-genome microarray chip (29 K), thus making it possible to profile approximately 29,000 genes (95\% of the predicted grapevine transcriptome). Applications available with the online platform include the use of gene names, probesets, modules or biological processes to query the co-expression networks, with the option to choose between Affymetrix or Nimblegen datasets and between multiple co-expression measures. Alternatively, the user can browse existing network modules using interactive network visualisation and analysis via CytoscapeWeb. To demonstrate the utility of the database, we present examples from three fundamental biological processes (berry development, photosynthesis and flavonoid biosynthesis) whereby the recovered sub-networks reconfirm established plant gene functions and also identify novel associations.

Conclusions: Together, we present valuable insights into grapevine transcriptional regulation by developing network models applicable to researchers in their prioritisation of gene candidates, for on-going study of biological processes related to grapevine development, metabolism and stress responses.

\section{Background}

The cultivated grapevine Vitis vinifera is one of the most highly valued horticultural crops in the world, and amongst the earliest domesticated fruit crops in human history. The global production of grapes in 2011 was 70 million tonnes, harvested over approximately 7 million hectares of land, making the grapevine the most widely cultivated fruit species [1]. Quality attributes of grapes, including aroma, flavour, colour and texture characteristics,

\footnotetext{
* Correspondence: christopher.ford@adelaide.edu.au

'School of Agriculture, Food and Wine, University of Adelaide, Adelaide 5064, South Australia, Australia

Full list of author information is available at the end of the article
}

have a profound impact on the fruit and wine and therefore on the value of the crop itself. An in-depth understanding of gene expression and the regulation of metabolic pathways controlling various aspects of grapevine development and berry metabolism could provide insights into the genetic factors influencing fruit quality and ultimately inform future vineyard germplasm and cultural practices.

Functional genomics studies in plants have contributed to a systems-level understanding of how genes function and how an underlying biological process is governed by the cooperation of a set of genes. Genome sequencing of two grapevine cultivars $[2,3]$ and successive improvements

\section{Biomed Central}


on genome assembly and prediction [4-6] have been invaluable for gene discovery, while application of high throughput technologies such as microarrays has enabled large-scale transcriptional analysis in grapevine. During the pre-genomic period, sequences selected from Genbank, expressed sequence tags and the NCBI RefSeq grapevine transcripts were the main sources for the design and annotation of the grapevine $16 \mathrm{~K}$ Affymetrix Genechip (http:// www.affymetrix.com), with approximately one third of the transcriptome represented on the array based on the $12 \mathrm{X} v 1$ gene annotation [6]. The grapevine $29 \mathrm{~K}$ Nimblegen whole-genome array, (http://www.nimblegen.com), which represents approximately 29,000 genes (>95\% of the predicted transcriptome) is the most well-developed of the grapevine microarray platforms. To date, microarray studies feature numerous experiments, including different stages of berry development for various cultivars $[7,8]$, a range of grapevine tissues $[9,10]$ and the application of various biotic and abiotic stressors [11,12]. A survey from gene expression data repositories including the Gene Expression Omnibus [13] and Arrayexpress [14] revealed that a large number of expression datasets have been generated from plants, especially Arabidopsis thaliana, Glycine max (soybean) and Oryza sativa (rice), and these also involve diverse experimental conditions. Although these gene expression datasets have been primarily generated within a particular experimental context, the accumulation of large numbers of expression profiles has offered additional capabilities. These include comparative genomics between plant species, screening and functional assignment of gene candidates, the discovery of novel DNA motifs, and the dissection of regulatory networks. One technique that has proved invaluable in this role is that of gene coexpression analysis (GCA).

GCA is based on the notion that genes involved in similar or related processes may exhibit similar expression patterns over a range of experimental conditions $[15,16]$. This "guilt by association" principle has been initially applied to gain insights into co-expressed gene modules within an organism $[17,18]$, to assign novel gene functions previously not ascribed to any biological processes, and to understand the evolution of gene expression and diversity across species and kingdoms $[19,20]$. A 'condition-independent' GCA derived from a large dataset compiled from various experimental conditions has been adopted in many studies for convenience while providing a global overview of gene-to-gene relationships [21-23]. However, drawbacks to the conditionindependent approach include the complexity of drawing biological insights and the potential loss of co-expression relationships due to variation between the numerous experimental conditions. Alternatively, the 'conditiondependent' approach, which draws upon GCA derived from smaller and predefined datasets (conditions), provides an additional opportunity to test specific hypotheses or to gain biological insights in an underlying condition [24,25]. However, when too few sample datasets are chosen, noise inherent in the microarray data will also affect the results obtained from 'condition-dependent' GCA. Nevertheless, both 'condition-independent' and 'condition-dependent' approaches have proven useful in many co-expression studies in plants [16].

Within a co-expression network, genes and similarity relationships (commonly represented by correlation coefficients) can be visualised as "nodes" and "edges" respectively. The connection of two nodes by an edge indicates a similar expression profile of the nodes according to a particular similarity metric. For a given set of genes, the collection of these nodes and edges forms a network. Visualisation of the co-expression network enables the identification and description of densely connected gene clusters, referred to as modules, and an assessment of biological relevance can be achieved by investigating the functions of genes within each module [15,26]. Many graph clustering algorithms have been developed with the aim of extracting functional modules comprising densely connected groups of nodes (representing coexpressed genes). Such algorithms can be classified as density-based and local search algorithms, hierarchical clustering, and other optimization-based algorithms [27]. In addition to the model plant Arabidopsis, these algorithms have also been applied to study co-expression networks in important crop species such as rice, barley and soybean [21,28], with databases developed to store inferred modules and provide a user-friendly resource for plant biologists. Examples of outcomes reported using the "guilt by association" principle include the identification of genes involved in cellulose biosynthesis [29] and transcription factors (TFs) involved in glucosinolate regulation in Arabidopsis [30].

In the present study, over 800 publicly available microarray datasets related to the $V$. vinifera L. transcriptome were selected to construct global co-expression networks (GCNs), consisting of 463 datasets from the Nimblegen whole-genome arrays and 403 datasets from the $16 \mathrm{~K}$ Affymetrix Genechip arrays. A combination of correlation rank transformation and graph-clustering approaches was used. With particular emphasis on the GCN constructed using the Nimblegen whole-genome array, we demonstrate the utility of this $V$. vinifera GCN using selected examples where we confirm well-characterised biochemical pathways, and infer potential novel gene functions and processes. A dedicated grapevine gene co-expression database, named VTCdb (http://vtcdb.adelaide.edu.au/Home. aspx), equipped with functional enrichment and visualisation capabilities, has been made available to the public to query and browse the associated GCN. 


\section{Construction and content Data acquisition and processing}

Publicly available grapevine $29 \mathrm{~K}$ NimbleGen wholegenome (http://www.nimblegen.com) and $16 \mathrm{~K}$ Affymetrix Genechip (http://www.affymetrix.com) microarray datasets were retrieved from Gene Expression Omnibus [13], Arrayexpress [14] and PLEXdb [31]. Summaries of the experiments and associated metadata are given in Additional files 1 and 2, detailing the 481 (Nimblegen) and 451 (Affymetrix) arrays (containing approximately 29,000 and 16,000 probesets respectively) that were used for subsequent analysis. Raw intensity data from both platforms were separately background-adjusted, quantilenormalised and summarised using the RMA method in R (http://www.r-project.org) using the 'oligo' package [32]. Potential outlier arrays were removed by visual inspection of raw perfect match data and iteratively discarding arrays that failed the quality control test (where expression values deviated significantly from the relative log expression and the normalised unscaled standard error) leaving 463 (Nimblegen) and 403 (Affymetrix) arrays for subsequent analysis (see Additional file 2). A survey of the underlying experimental conditions represented by the arrays can be assembled into a general category ('All' datasets) covering a broad range of treatments and plant development stages such as tissue development, stress and vineyard management strategies. Additionally, two condition-specific datasets were established for the $29 \mathrm{~K}$ Nimblegen datasets, one for berry-related tissues and treatments only, and one for stress-related processes (biotic and abiotic) across the whole vine (Additional file 2). The number of arrays corresponding to arrays for 'All', 'Berry' and 'Stress' datasets are 463, 305 and 59, respectively. Together, this provided a broad basis for inferring both condition- independent and dependent gene co-expression relationships in grapevine. Separate GCNs were generated for all, berry- and stress-related datasets by applying the procedure below.

\section{Gene co-expression network construction}

Correlations between all mapped probesets were calculated using Pearson's correlation coefficient (PCC) and Spearman's correlation coefficient (SCC) as measures of similarity between expression profiles. Additionally, the mutual co-expression relationships between all gene pairs were calculated (without applying any cut-offs) by first transforming raw correlation values (PCC and SCC) into highest reciprocal ranks (HRR) [33] and mutual ranks (MR) [34]. Rank-based networks are robust and offer advantages over correlation-based networks [34,35]. Such approaches have been frequently applied to retain weak but significant co-expression relationships and circumvent the unequal distribution of gene correlations for some genes when applying a fixed similarity threshold.
This index of co-expression (HRR and MR) serves as a basis for ranking co-expressed genes when using a guide gene' approach to query the network. In this study, we focussed our attention on the mutual co-expression relationships derived from PCC values for simplicity, and because the Gene ontology (GO) prediction performance of transformed ranks from PCC and SCC values were similar [34]. An estimation of the statistical significance of mutual co-expression ranks [21] showed that HRR and $M R$ values $\leq 350$ and 200 respectively were significant $(P<0.01)$, and therefore these were applied as a generalised threshold for obtaining biological relevant relationships in grapevine.

\section{Graph clustering and meta-network construction}

To identify modules of densely connected nodes, the Heuristic Cluster Chiselling Algorithm (HCCA) [33] and Markov clustering (MCL) [36] techniques were applied. With an input network of $\mathrm{HRR} \leq 30$, we first assigned weights of $0.2,0.067$, and 0.04 to HRR scores of 10, 20 and 30 , respectively. Parameters were adjusted to a desired step size of 3 and cluster size between 40 and 400 for HCCA and an inflation value of 1.2 for MCL using Python 2.7.3 (http://www.python.org) and MCL version 12-068 (http://micans.org/mcl/), respectively. To depict the relationships between modules generated, we first calculated the total edge weights shared between any two connected modules and assigned an empirically derived statistical significance ( $P$-value) by permutation test according to [21]. The various grapevine metanetworks were constructed with edges connecting modules at a significance of $P<0.01$.

Functional enrichment and expression specificity analysis To assist with the categorisation of co-expressed genes and partitioned modules according to their potential function or processes, we assessed the modules for enrichment primarily for GO terms in R (http://www.rproject.org) using the 'gProfileR' package [37] to interface g:profiler (http://gprofiler.at.mt.ut.ee/gprofiler/). Enrichment for GO terms was validated using the hypergeometric distribution adjusted by set count sizes (SCS) for multiple hypothesis correction. SCS threshold considers the hierarchal structure of GO in an underlying organism and prioritises truly significant results (while removing enriched false positive GO terms) [37]. GO terms were considered significant if the adjusted $P$-values $(\mathrm{SCS})<0.05$ and there were at least two genes associated with the same annotation. Network representation of GO terms was prepared using GO-module webserver [38]. Expression specificities of individual probesets and modules were determined using the Std2Gx procedure [28]. Expression specificity index values $>1$ and $>5$ indicates the gene is well and specifically expressed in the corresponding 
experimental condition respectively, as compared with other genes and array samples. Expression specificity of modules is expressed as the percentage of module members specifically expressed in a particular tissue/condition (and across all arrays) with an expression specificity index above 1 .

\section{Gene annotations and network visualization}

The latest grapevine gene and probeset annotations based the $12 \mathrm{X}$ v1 prediction were obtained from Vitisnet $[4,39]$ and mappings for probesets containing functional annotation and categorization of predicted genes (chromosome location, predicted function, subcellular localization, orthology and pathway level information) were used. CytoscapeWeb [40] was used to visualise nodes and edges and their attributes.

\section{Utility}

\section{VTCdb web interface and content}

VTCdb (http://vtcdb.adelaide.edu.au/Home.aspx) can be accessed via a user-friendly web interface that includes tools to query, browse and visualise the co-expression network genes and modules. VTCdb runs on an Internet Information Server (IIS, version 7.0) containing data tables stored in MS Access (Microsoft Corporation Inc., USA). The web pages were built using a combination of ASP.NET 4.0, Javascript and Jquery 1.4. The VTCdb home page contains several search forms to retrieve co-expressed genes and related information, including a co-expressed genes search, a keyword search, an enriched term search, and an option to browse the metanetwork interfaces (Figure 1A-E). With the current release, queries can be performed against the grape co-expression network inferred using the Nimblegen grape genome arrays (default) or the grape Affymetrix Genechip arrays.

Under the single guide gene query, when a gene identifier (i.e. VIT_ code) is used as a query using the 'CoexQuery' field, the user can select the various predefined conditions ('All', 'Berry' and 'Stress') followed by the preferred co-expression measure ('HRR', 'MR' and 'CORR') (Figure 1B). Users will be re-directed to the co-expressed genes result page, 'CoexQuery result' for the chosen gene, with the chosen dataset and co-expression measure.

To search for a term or description associated with a gene, users should input the keyword of interest and select the 'keyword' option (Figure 1C). The keyword query tool will perform a broad search across various fields (i.e. gene identifiers, symbols, functional annotations, associated ontology/pathway terms) within the gene annotation tables for matches and redirects users to the 'keyword results' page containing associated genes, information and links for further downstream analysis using the various toggles (Figure 2A). To search for enrichment descriptors (GO ID or term; if present) within sub-networks of guide-genes and modules, select the 'enriched term' button (Figure 1D). Users will be redirected to the 'enriched keyword' page which provides an interface with associated links to the 'enrich keyword query' results page (Figure 2B). The 'enrich guide' (Figure 2C) and 'enrich module' (Figure 2D) results pages contain lists of enrichment values for the given $\mathrm{GO}$ term of interest and links to the 'CoexQuery' and 'module results' pages, respectively. Checkboxes allow users to choose between these various parameters. Additionally, via the CytoscapeWeb interface, users are able visualise meta-networks (i.e. All_HRR_MCL) and browse nodes (modules in this case) within the meta-network (Figure 1E). By moving the cursor over the module of interest, significantly connected modules become highlighted and details of the module size, number of edges and enriched GO BP terms are given. Double clicking the modules takes users to the module result page, with detailed information on the module of interest.

The co-expressed genes 'CoexQuery' result page contains a table with detailed information (gene annotation) on query genes and the associated module (when identifiable using $\mathrm{HCCA}$ or $\mathrm{MCL}$ ) to which the query gene belongs (Figure 3A). Next, a list containing information on co-expressed genes (ranked in ascending order of coexpression strength of desired condition) is displayed (Figure 3B). In brief, the table displays the top 50 coexpressed genes, links to 'CoexQuery' result pages for each gene, and the co-expression strength in the conditions of interest (i.e. 'all', 'berry' and 'stress' networks, where applicable). Insignificant ranks (HRR > 750, MR > 550; $P>0.05)$ in corresponding conditions are coloured in grey. Using various checkboxes, the table can be fully expanded to show all significant co-expressed genes and additional annotations (i.e. predicted functional annotation, localization, and molecular network). Clicking on a sub-column in the 'CoexQuery' field (i.e. 'all', 'berry' or 'stress') in any row will then open a new 'CoexQuery' result page with the selected network for the corresponding gene. Columns can be sorted by clicking the headers of the table, which provides flexibility for the user interested in ranking the co-expressed genes list according to other conditions of interests (Figure 3C), indexes of co-expression (e.g. PCC) or grouping molecular network annotations. Functional (GO) category overrepresentation of co-expressed genes will be displayed (if present) in a table sorted in ascending enrichment $P$-values for every GO category along with test statistics for the enriched $\mathrm{GO}$ term and information of the enriched genes (Figure 3D). Alternatively, users can submit the list of co-expressed genes to gprofiler for functional enrichment analysis. Expression specificity of the query gene in various experimental conditions is displayed 


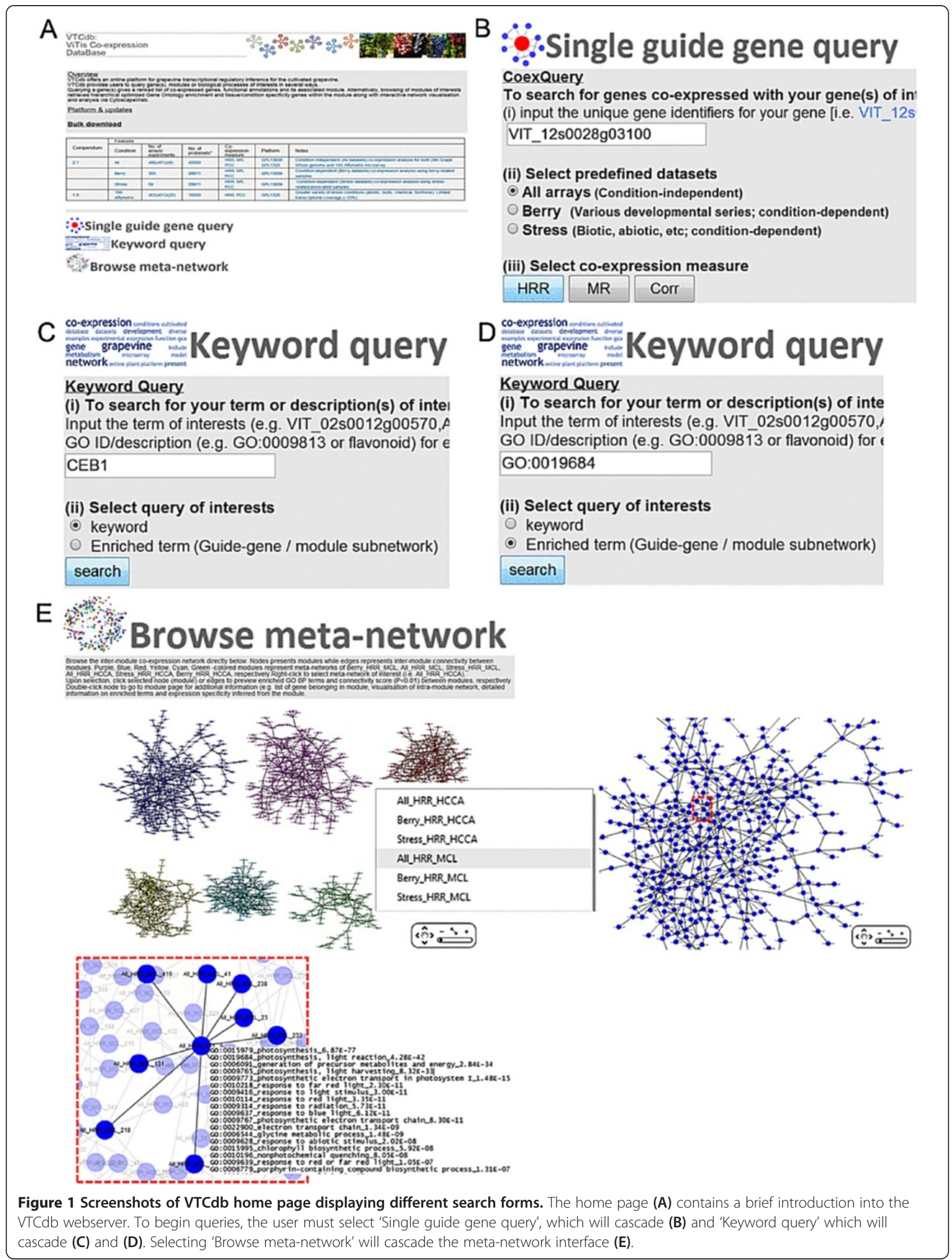


A $\square$ Hide/show links to CoexQuery and ModuleQuery functions [Hide/show additional information on query results (genes) \begin{tabular}{llll|}
\hline Platform & ProbeID & Symbol & Functional annotation \\
$29 \mathrm{~K}$ & VIT_01s0244g00010 CEB1 & Cell elongation bHLH (CEB1)/BHLHDOM_53
\end{tabular}

VHide/show links to CoexQuery and ModuleQuery functions $\nabla$ Hide/show additional information on query results (genes)

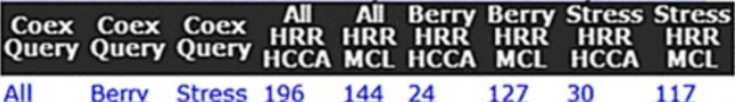

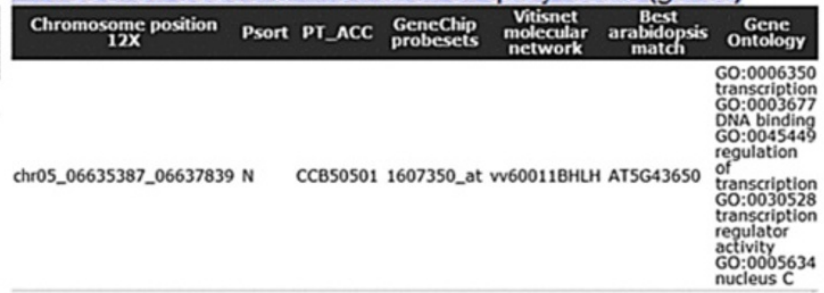

B Hide/show links to enrichguide query functions

$\checkmark$ Hide/show links to enrichmodule query functions
GO ID
GO type
GO description
enrichguide

GO:0019684 BP

photosynthesis, light reaction Guide

\begin{tabular}{|ccc|}
\hline GO ID GO type & GO description & $\begin{array}{c}\text { enrichmodule } \\
\text { Query }\end{array}$ \\
\hline
\end{tabular}

GO:0019684 BP photosynthesis, light reaction Module

C Condition: All, CR: HRR; Hide/show links to CoexQuery functions

$\square$ Condition: Berry, CR: HRR; Hide/show links to CoexQuery functions

$\square$ Condition: Stress, CR: HRR; Hide/show links to CoexQuery functions

Condition: All, CR: MR: Hide/show links to CoexQuery functions

Condition: Berry, CR: MR; Hide/show links to CoexQuery functions

$\square$ Condition: Stress, CR: MR; Hide/show links to CoexQuery functions

DShowhide 16K Affymetrix probesets

adjp-
value GO ID $\begin{gathered}\text { GO } \\ \text { type }\end{gathered}$

6.89E-50 GO:0019684 BP photosynthesis, vit 0350088g01190 PMDH2

\begin{tabular}{|c|c|c|}
\hline $1.75 E-49$ & GO:0019684 BP & $\begin{array}{l}\text { photosynthesis, viT 09s0002004320 PSI-H } \\
\text { light reaction }\end{array}$ \\
\hline $5.96 E-49$ & GO:0019684 BP & $\begin{array}{l}\text { photosynthesis, VIT } 1150065001100 \text { PPR } \\
\text { light reaction }\end{array}$ \\
\hline $7.33 E-47$ & GO:0019684 BP & $\begin{array}{l}\text { photosynthesis, vIT 04s0023000410 PSAL } \\
\text { light reaction }\end{array}$ \\
\hline $1.27 E-46$ & GO:0019684 BP & $\begin{array}{l}\text { photosynthesis, ver } 0250012001120 \text { glycine cleavage system } \mathrm{H} \text {, } \\
\text { might reaction }\end{array}$ \\
\hline
\end{tabular}

Malate dehydrogenase, glyoxysomal precursor

D Condition: All, CR: HRR, GC: HCCA; Hide/show links to ModuleQuery functions

$\square$ Condition: Berry, CR: HRR, GC: HCCA; Hide/show links to ModuleQuery functions

$\square$ Condition: Stress, CR: HRR, GC: HCCA; Hide/show links to ModuleQuery functions

Condition: All, CR: HRR, GC: MCL; Hide/show links to ModuleQuery functions

Condition: Berry, CR: HRR, GC: MCL; Hide/show links to ModuleQuery functions

Condition: Stress, CR: HRR, GC: MCL; Hide/show links to ModuleQuery functions

\begin{tabular}{|l|lllll}
\hline \multicolumn{2}{|c}{ adj p-value } & GO ID & GO type & GO description & $\begin{array}{c}\text { Module } \\
\text { Query }\end{array}$ \\
\hline 41 & $4.28 \mathrm{E}-42$ & GO:0019684 BP & photosynthesis, light reaction 6 \\
\hline 10 & $1.13 \mathrm{E}-10$ & GO:0019684 BP & photosynthesis, light reaction 21 \\
\hline 4 & $4.24 \mathrm{E}-05$ & $\mathrm{GO}: 0019684$ & $\mathrm{BP}$ & photosynthesis, light reaction 233 \\
\hline 2 & 0.0254 & GO:0019684 BP & photosynthesis, light reaction 133 \\
\hline
\end{tabular}

Figure 2 (See legend on next page.) 
(See figure on previous page.)

Figure 2 Screenshots of VTCdb pages relevant to the utility and output of keyword query, enrich guide and enrich module searches. The 'Keyword' result page (A), includes probesets and annotations with matching terms, as well as links to the 'Co-expressed genes' and 'Module' results pages. The 'Enrich keyword query' result page (B) reports matching GO query terms (ID/ description) and provides links to the 'Enrich guide' (C) and 'Enrich module' (D) results pages. The 'Enrich guide' result page (C) reports genes in which their co-expressed genes are functionally enriched with GO query terms of interests. The page contains checkboxes to select criteria of interest and a table contains functional annotations of genes, enrichment values of GO query terms and gene links to perform 'Single guide gene query' with the selected parameters of interest. Similarly, the 'Enrich module' results page (D) reports modules in which genes within the module are functionally enriched with GO queries of interest. The page contains checkboxes to select criteria of interest and a table containing enrichment values of GO query terms and module links to perform 'Module query' with the selected parameters of interest.

in a graph format (Figure 3E). By moving the cursor over the graphs, users can see the underlying experimental conditions and expression specificities of respective modules. Alternatively, the expression specificity table for the guide gene can be viewed when the hide/show expression specificity is checked. A network representation of the top 10 co-expressed genes with the query (guide) gene from various predefined datasets can be visualised using Cytoscapeweb (Figure 3F). Additional information on annotations and co-expression conditions can also be viewed by placing the cursor over the nodes or edges, respectively. In addition, users can view the top 10 co-expressed genes for a condition-dependent network by right-clicking and selecting the preferred condition. Similarly, the 'Module result' page (Figure 4) is composed of four separate tables, the first containing genes belonging to the module (Figure 4A), the second containing GO enriched terms (sorted by type and SCS significance) (Figure 4B), the third containing expression specificity of the inferred module (Figure 4C), and the final table is a list of significantly connected modules (Figure 4D). Again, various checkboxes can be used to hide/expand additional information pertaining to genes, modules and enrichment terms. The expression specificity graph allows the user to visualise tissue/sample conditions under which the majority of the probesets within that module are specifically expressed (Figure 4C). Additionally, network visualisation tools for networks analysis are provided using the CytoscapeWeb application (Figure 4E). Functions such as displaying node/edge annotations, highlighting first-neighbours of nodes, and visualisation at different cut-off parameters enable manipulation of the co-expression network according to user preference.

\section{Discussion}

To demonstrate the applicability and robustness of the VTCdb web server for co-expression studies, we present some examples for the use of VTCdb query tools in the analysis of well-characterised biological processes and highlight gene co-expression networks that may be of biological interest in future grapevine research.

\section{Example application I: grapevine berry development}

Grapevine fruit development can be broken into 3 phases by chronological sequence: berry formation, veraison and berry ripening, reviewed in [41]. Each of these involves specific changes in gene expression, biochemical, compositional and physiological properties of the berry. For example, processes involving cell wall reorganization are crucial during periods of rapid cell division and elongation (during berry formation) and softening (during berry ripening). Accumulating evidence suggests that the involvement of various activities of grapevine expansins (among others) are crucial in regulating cell wall expansion and enlargement during berry development [42-44]. To provide additional insights into the transcriptional regulation of cell wall metabolism during grapevine development, co-expression analysis using a grapevine bHLH $\mathrm{TF}$, grapevine CEB1, which is known to regulate grape berry development [42] was performed. In this example, the respective unique code for grapevine CEB1 (VIT_01 s0244g00010) was input into the 'CoexQuery' field and selected 'all' and 'HRR' for the preferred predefined datasets and co-expression measure options, respectively (Figure 1B) or by using the keyword query 'CEB1' (Figure 1C) and choosing 'all' under the 'CoexQuery' column in the 'keyword query' results page. A total of 266 genes were indicated to be co-expressed with grapevine CEB1, with average HRR and PCC values of 167 and 0.73 respectively. Among others, genes encoding enzymes involved in auxin signalling (SAUR9, VIT_04s0023g03230; ARF2_2, VIT_01 s0244g00150; TPR1, VIT_04s0008g06400), cell wall metabolism (EXPA11, VIT_18s0001g01130) and various classes of TF (bHLH, ERF, MYB) were highly co-expressed with grapevine $V v C E B 1$ (Additional file 3: Table S1). In agreement with the co-expression results, experimental evidence has shown that overexpression of VvCEB1 in grapevine embryos is able to stimulate cell expansion via control of Auxin/IAA TFs, SAUR and cell wall modification genes [42]. Interestingly, among the transcripts tested, EXPA11 (VIT_18s0001g01130, XM_002285855.1 in their study) was the most up-regulated ( $>1000$ fold) in VvCEB1overexpressing grape embryos compared to control [42]. In the co-expressed genes results, EXPA11 (VIT_18 s0001g01130) was highly co-expressed (top 6) with grapevine $V \nu C E B 1$ in 'all' conditions while the HRR (top 1) were 


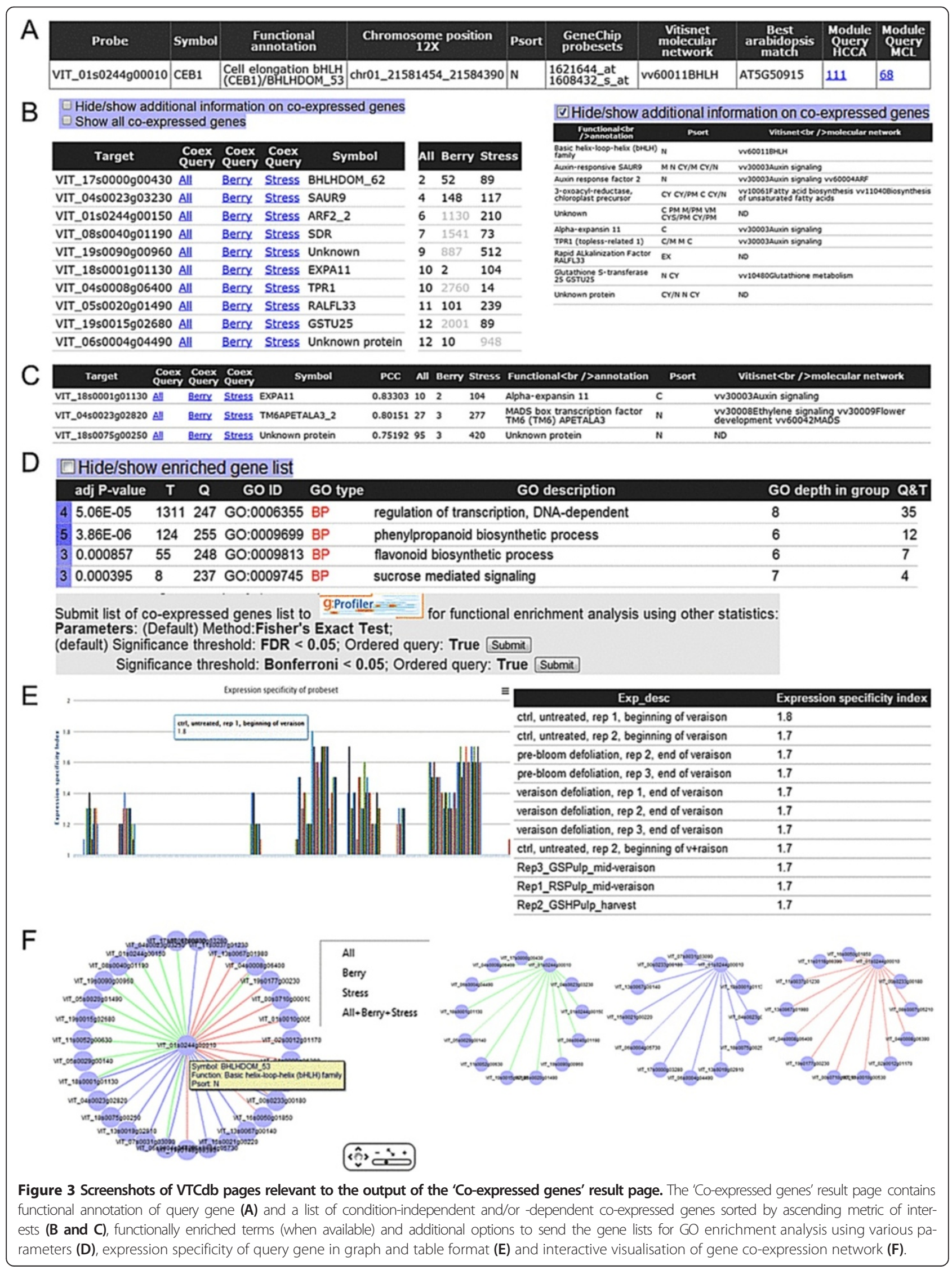


A 0 Hide/show additional information on co-expressed genes

Show all genes within module

\begin{tabular}{|c|c|}
\hline Probeld & Functional annotation \\
\hline VIT_0150010903620 LHCA2 & $\begin{array}{l}\text { LHCA2 (Photosystem I } \\
\text { light havestosting complex } \\
\text { gene 2) }\end{array}$ \\
\hline VIT_00s0181g00180 LHCB3*1 & $\begin{array}{l}\text { LHCCB3 (IIphthanesting } \\
\text { chlorophyil binding protein } \\
\text { 3) }\end{array}$ \\
\hline VIT_00s0181g00200 LHCB3*1 & $\begin{array}{l}\text { LHCB3 (light-havesting } \\
\text { chlorophyil binding protein } \\
\text { 3) }\end{array}$ \\
\hline VIT_03s0088901190 PMDH2 & $\begin{array}{l}\text { Malate dehydrogenase. } \\
\text { glyoxysomal precursor. }\end{array}$ \\
\hline VIT_01s0011g02070 hydrolase & $\begin{array}{l}\text { Metal-dependent } \\
\text { phosphohydrolase HD } \\
\text { domain-containing protein }\end{array}$ \\
\hline VIT_01s0127900420 ECHID & Naphthoate synthase \\
\hline VIT_02s0109g00080 Phosphonbulokinase & Phosphorbulokinase \\
\hline VIT_00s0125g00280 PSAF & $\begin{array}{l}\text { Photosystem I reaction } \\
\text { center subunit ifi (PSAF) }\end{array}$ \\
\hline VIT_0150137g00210 YCF32 & $\begin{array}{l}\text { Photosystem II core } \\
\text { complex proteins psby, } \\
\text { chloroplast precursor }\end{array}$ \\
\hline VIT_00s0904g00010 PSBQ & $\begin{array}{l}\text { Photosystem II oxygen. } \\
\text { evoling enhancer protein } \\
\text { PSBQ }\end{array}$ \\
\hline
\end{tabular}

\section{VHide/show additional information on co-expressed genes}

\begin{tabular}{|c|c|c|}
\hline 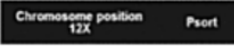 & 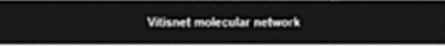 & $\begin{array}{l}\text { ardest } \\
\text { anteh }\end{array}$ \\
\hline 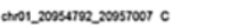 & 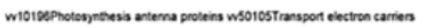 & ATJGE1470 \\
\hline AMm_07600002_-07831816 C & 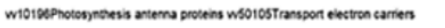 & ATSOS4270 \\
\hline Anm_07es5106_07868115 C & 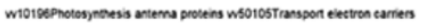 & ATSOS4270 \\
\hline 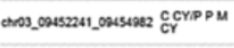 & 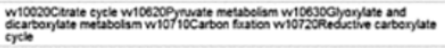 & ATSOOSese \\
\hline Ctro1__01751432_01780010 PNPM & No & AT1G1330 \\
\hline etror__ensesor_onsor11 MCM & 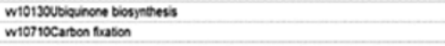 & $\begin{array}{l}\text { AT16eosso } \\
\text { No }\end{array}$ \\
\hline 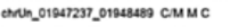 & 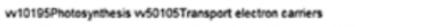 & AT1031330 \\
\hline 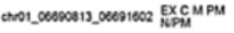 & 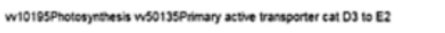 & ATIGETVOS \\
\hline & 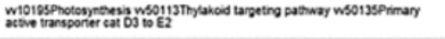 & 10 \\
\hline
\end{tabular}

B adj P.value I Q GO ID GO type

\begin{tabular}{|c|c|c|c|c|c|c|}
\hline 76. & $6.87 \mathrm{E} \cdot 77$ & 149 & $240 G 0: 0015979$ GP & photosynthesis & 4 & 60 \\
\hline 41 & 4.26E-42 & 80 & $240 \mathrm{GO}: 0019684 \mathrm{BP}$ & photosynthesis, light reaction & 5 & 33 \\
\hline 11 & $3.38 E-116$ & 391 & $240 \mathrm{GO}: 0009579 \mathrm{CC}$ & thylakoid & 5 & 104 \\
\hline 112 & $1.5 E-112$ & 878 & $240 \mathrm{GO}: 0044434 \mathrm{CC}$ & chloroplast part & 9 & 129 \\
\hline 7 & 1.25E-07 & 4 & $240 \mathrm{G0}: 0003959 \mathrm{MF}$ & NADPH dehydrogenase activity & 5 & 4 \\
\hline 7 & $1.26 \mathrm{E}-07$ & 16 & $240 \mathrm{GO}: 0008187 \mathrm{MF}$ & poly-pyrimidine tract binding & 7 & 6 \\
\hline
\end{tabular}

Submit list of co-expressed genes list to g-Profiler - for functional enrichment analysis using other statistics: Parameters: (Default) Method:Fisher's Exact Test;

(default) Significance threshold: FDR $<0.05$; Ordered query: True Submat Significance threshold: Bonferroni $<0.05$; Ordered query: True Submit

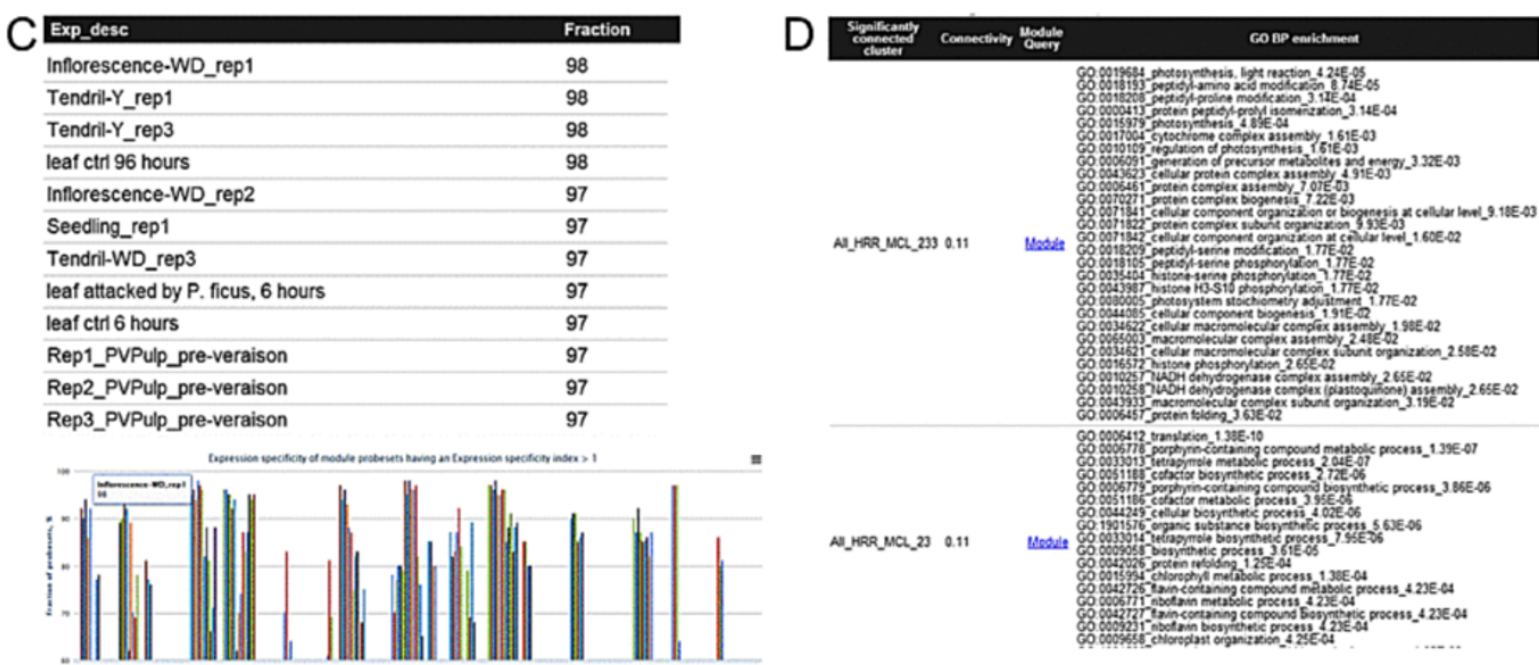

E
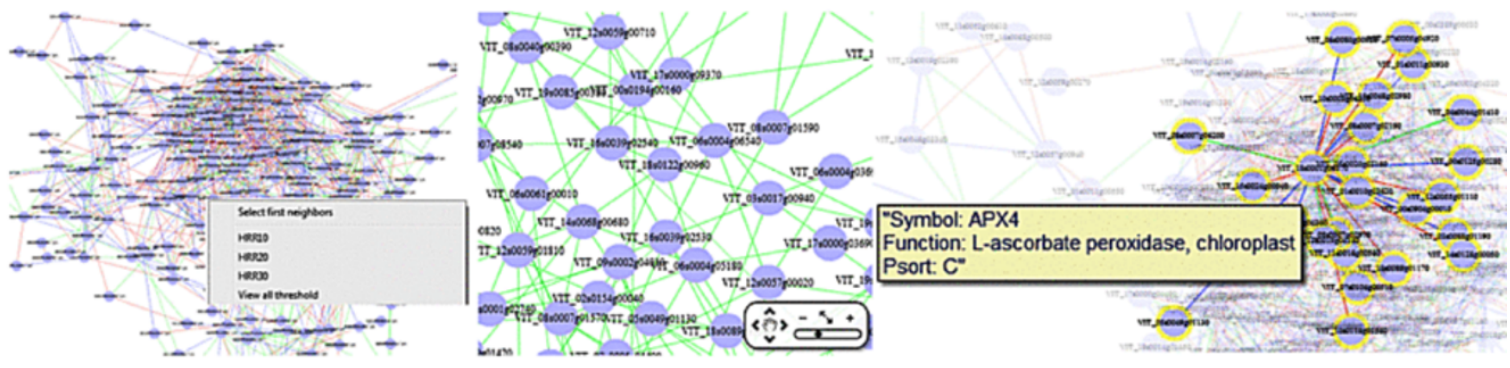

Figure 4 (See legend on next page.) 
(See figure on previous page.)

Figure 4 Screenshots of VTCdb pages relevant to the output of the 'Module' result page. The 'Module' results page contains a list of genes belonging to the module (A), functionally enriched terms (when available) and additional options to send the gene lists for GO enrichment analysis using various parameters (B), expression specificity of genes belonging to associated module in graph and table format (C), a list of significantly connected modules with query module (D) and interactive visualisation of gene co-expression network (E).

improved in berry-related datasets (Figure 3C). As an alternative, selecting other predefined conditions to understand the molecular mechanisms of query genes in a specific context could also be carried out. Nevertheless, using 'all' datasets is sufficient for most applications. Below the list of co-expressed genes, GO enrichment analysis of the whole co-expressed gene lists ( $\mathrm{HRR}<350, P<0.01)$ revealed that terms such as GO:0006355, regulation of transcription DNA-dependent (5.06e-05); GO:0009699, phenylpropanoid biosynthetic process (3.86e-06); GO:0009813, flavonoid biosynthetic process (8.57e-04) and GO:0009745, sucrose mediated signalling (3.95e-04) were highly enriched (Figure 3D; Additional file 3: Table S2). This data suggests the potential involvement of additional TFs, phenylpropanoid (shikimate) pathway genes and sucrose metabolism in regulating the cell expansion during berry development. Additionally, the highly interactive expression specificity chart showed that the grapevine $V v C E B 1$ gene was expressed specifically in berry-related tissues with highest specificity during veraison onwards (Figure 3E; Additional file 3: Table S3). Taken together, the coexpression results largely confirm results from previous studies and strengthen the putative role of $V \nu C E B 1$ in controlling berry growth while providing additional clues into the complex molecular mechanisms of VvCEB1 [i.e. potential targets (direct/indirect), expression specificity and enriched pathways].

\section{Example application II: photosynthesis and phenylpropanoid metabolism}

When a priori knowledge of a target gene is not known, searches using terms of interest enriched within predicted modules can be conducted using VTCdb. Users can query broad GO terms (ID/description) such as GO:0019684 or 'photosynthesis, light reaction' (Figure 1D) and select the preferred condition and graph clustering approach in the 'enrich module results' page (Figure 2D). In this example, when a condition-independent (all) and MCL approach is selected, modules (ALL_MCL) 6, 21, 233 and 133 were enriched with GO:0019684 (photosynthesis, light reaction) with module All_MCL_6 having the highest adjusted $P$-values of 4.28E-42. Upon browsing All_MCL_6, it could be seen that the module showed 240 nodes, of which 68 had predicted roles related to photosynthesis $(\sim 30 \%)$, while some were involved in antioxidant detoxification and were predicted components of the chloroplast ascorbate-glutathione cycle [45] (Figure 5A). Such genes included those encoding chloroplastic ascorbate peroxidase (VIT_18s0001g06370 and VIT_04s0008g05490), peroxiredoxin (VIT_11s0016g 00560), thioredoxin (VIT_18s0001g00820, VIT_18s0001g 10510 and VIT_18s0001g15310) and glutathione Stransferase (VIT_06s0004g03690). Furthermore, genes encoding proteins involved in electron transport, tetrapyrrole metabolism, the pentose phosphate pathway, glycine/ serine cleavage system and vitamin metabolism were clustered with photosynthesis-related genes (Figure 5A, Additional file 3: Table S4). As anticipated, truly significant and highly enriched ontological terms for biological processes (GO: BP) such as GO:0015979, photosynthesis (6.87E-77); GO:0015995, chlorophyll biosynthetic process (5.92E-08); GO:0009773, photosynthetic electron transport (8.30E-11) and GO:0006544, glycine metabolic process (1.48E-09) were highly enriched within this module (Figures 4B and 5B, Additional file 3: Table S5). This observation is consistent with the many co-expression studies previously conducted in Arabidopsis, where genes involved in photosynthesis and related processes were found to form well-defined co-expression modules [46]. This is likely because photosynthesis requires the coordinated assembly of proteins into large super-complexes with numerous protein-protein interactions, and therefore their corresponding genes are expected to be highly coexpressed [47]. Interestingly, the sub-network of a putative grapevine GUN4 (VIT_05s0102g00310) was connected to genes encoding proteins involved in photosynthesis (i.e. LHCB6, VIT_12s0055g01110; LHC2 type 1, VIT_10s 0003g02900) and tetrapyrrole metabolism (CHLD, VIT_06 s0061g00010), corroborating the role of GUN4 in regulating photosynthesis and chlorophyll development at the post-translational level [48] (Figure 5A). Additionally, a node annotated as a putative APRR2 (VIT_02 s0012g00570) within this module was connected to several photosynthesis-related genes (LHB1B1, VIT_12s0028g 00320 and PSBW, VIT_14s0081g00060) and vitamin E metabolism (VTE5, VIT_13s0074g00040 and PSY, VIT_04 s0079g00680) which was in agreement with the functional role of APRR2 in co-regulating genes primarily involved in photosynthesis and chloroplast functions, resulting in increased plastid size, chlorophyll content and pigmentation in plants [49] (Figure 5A). While the majority of nodes within this module were successfully annotated, there are several nodes in which no clear annotation has been ascribed by homology searches and which can nonetheless be hypothesized to be involved in photosynthesis-related functions given their tight co-expression relationships 


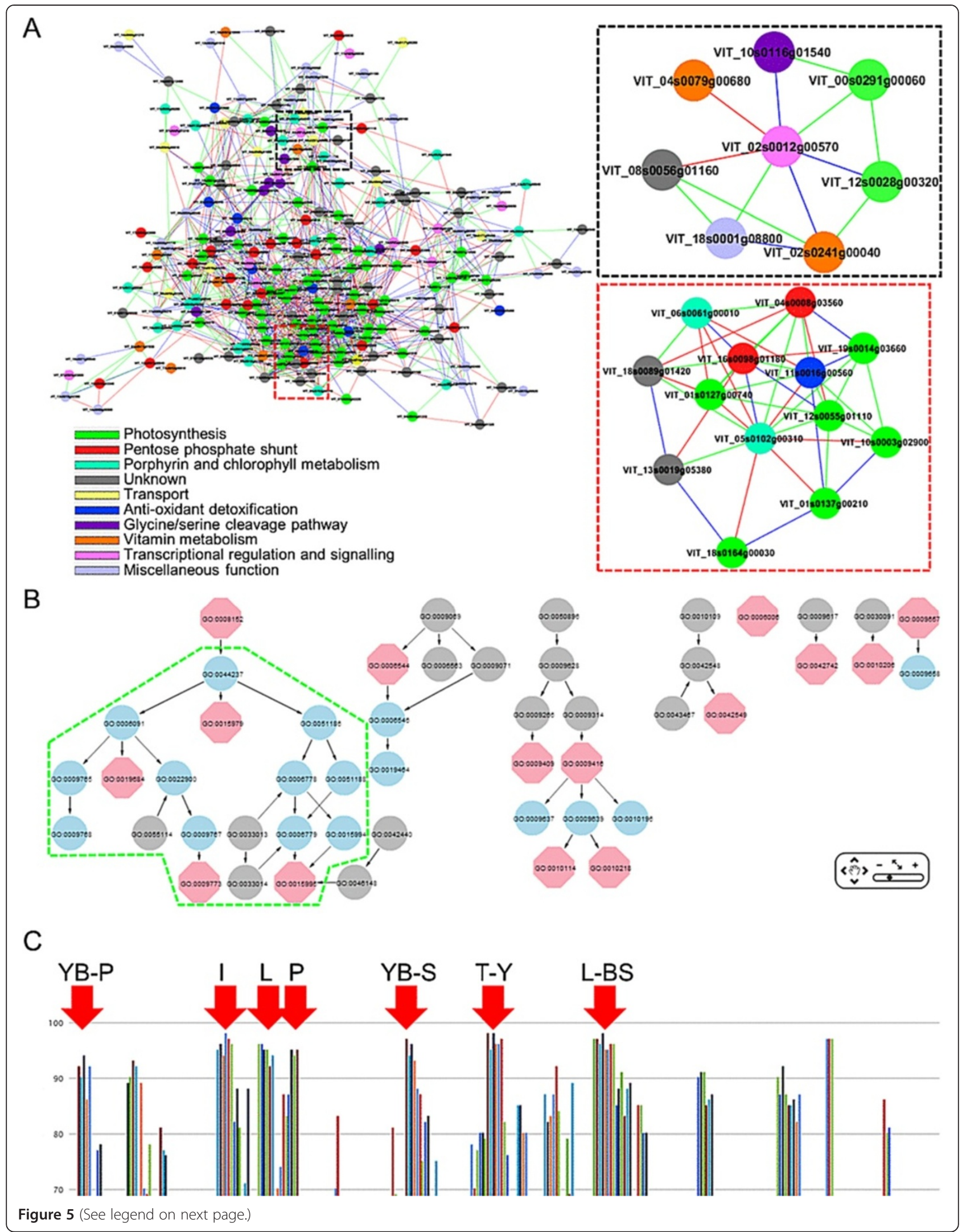


(See figure on previous page.)

Figure 5 Predicted module involved in photosynthesis (Module All_MCL_6). (A) Output for module 'All_MCL_6', which Contains 240 nodes and 1419 edges, with genes encoding proteins predominantly involved in photosynthesis (green), pentose-phosphate pathway (red), porphyrin and chlorophyll metabolism (cyan), transport (yellow), anti-oxidant detoxification (blue) and vitamin metabolism (orange) while others were involved in glycine/serine cleavage pathway (purple), transcriptional regulation and signalling (pink) and miscellaneous functions (light blue). Grey nodes represent genes whose function remains unknown or uncharacterised based on homology searches. Sub-network of putative APRR2 and GUN4 extracted from module 'All_MCL_6' within a neighbourhood distance of 1 (i.e. nodes connected directly to the query gene) are highlighted in black and red boxes, respectively. (B) Network representation of truly significant and highly enriched GO:BP terms related to photosynthesis, electron transport and chlorophyll metabolism are highlighted in green boxes. Other enriched GO terms were removed from presentation for clarity. (C) Expression specificity of module 'All_MCL_6' showing experiments in which at least 70\% of the probesets demonstrated specific expression (Std2Gx > 1.0). YB-P, young berries (pericarp); I, inflorescence; L, leaves; P, petals; YB-S, young berries (skin); T, tendrils (young); L-BS, leaves-biotic stress.

(Figure 5A) [50]. The expression specificity of All_MCL_6 also indicated that genes within the module were specifically expressed in leaves, seedlings, tendril, inflorescence and young berries, while expression levels were very low in post-veraison berries, seeds and callus samples (Figures 4C and 5C, Additional file 3: Table S6). This is not unexpected, considering their functional roles in photosynthesis, and their association with the chloroplast and photosynthetic tissues. At the cluster level (metanetwork), module All_MCL_6 was significantly connected $(P<0.01)$ to many modules including (All_MCL) 238, 389, 156, 416, 233 and 23 (Additional file 3: Table S7). GO BP functional analysis of the latter modules found that they were highly enriched with translation, redox metabolism, photosynthesis and tetrapyrrole metabolism terms. Similar to the enrichment of biological processes within modules, connected modules may participate in related processes given they share a significant proportion of coexpressed gene pairs and may provide additional information on the organization and coordination of module within the meta-network of co-expressed genes. From these data, it seems likely that genes constituting module All_MCL_6 are involved in the maintenance of redox regulation and homeostasis in the chloroplast thylakoids during photosynthesis and related processes.

To search for interesting modules involved in phenylpropanoid metabolism, a query using broad keyword terms such as "GO:0009698" or "phenylpropanoid metabolic process" and selecting the preferred condition (all datasets and HCCA clustering), returned a list of modules in which the query term was enriched (Additional file 3: Figure S1). Further inspection showed that members within respective modules (HCCA) were involved in various specialised pathways downstream of the main phenylpropanoid pathway, such as stilbenoid biosynthesis (module All_HCCA_60), flavonoid biosynthesis (module All_HCCA_181), lignin/lignan metabolism (modules All_HCCA_65 and All_HCCA_139), and the hypersensitive response (modules All_HCCA_131 and All_ HCCA_186). Natural products derived from the phenylpropanoid pathway play various fundamental roles in plants, including protection against abiotic stress, plantpathogen/herbivore interaction and plant development.
These secondary metabolites can encompass various classes of anthocyanins, flavonols, proanthocyanidins, lignins, terpenes and stilbenes. In this example we describe, in greater detail, how useful information could be obtained from module All_HCCA_181 (Figure 6). This module contained 74 nodes and 152 edges, represented predominantly by structural genes encoding enzymes of the flavonoid biosynthetic pathway (flavonone 3-hydroxylase, VIT_04s0023g03370; dihydroflavonol 4-reductase, VIT_18s0001g12800; leucoanthocyanidin dioxygenase, VIT_02s0025g04720), shikimate pathway (shikimate dehydrogenase, VIT_14s0030g00650; chorismate mutase, VIT_14s0108g01330; 3-deoxy-D-arabinoheptulosonate 7-phosphate synthase, VIT_00s0391g00070), TFs (MYBPA1, VIT_15s0046g00170) and transferase/ transport proteins (Transparent testa 12, VIT_12s0028g 01150) (Figure 6A, Additional file 3: Table S8). GO enrichment showed that GO:BP terms such as flavonoid biosynthetic process (GO:0009813; SCS $<2.14 \mathrm{e}-10$ ) and aromatic amino acid family metabolic process (GO:0009072; SCS < 6.54e-06) were significantly enriched as anticipated (Figure 6B; Additional file 3: Table S9). Furthermore, the sub-network surrounding the grapevine TF MYBPA1 in module All_HCCA_181 showed that the TF was linked to structural genes of the flavonoid metabolic pathway, corroborating previous gene expression and promoter studies [51,52] (Figure 6A). The majority of genes from module All_HCCA_181 (>70\%) were specifically expressed in berry skins (during véraison and ripening), flowers, buds, inflorescence, buds and rachis (FS and PFS) coinciding with the tissues and developmental programming of flavonoid accumulation [53] (Figure 6C, Additional file 3: Table S10). Additionally, module All_ HCCA_181 was significantly connected $(P<0.01)$ to modules (All_HCCA) 13, 90, 60, 242 (Additional file 3: Table S11). Functional analysis (GO:BP) of the latter modules suggested that they were highly enriched with ontologies involved in fatty acid (lipids, steroids, wax), hormone, anthocyanin and stilbenoid metabolic processes. Also, there was enrichment for genes involved in stress responses, which taken together suggests a strong coordination between modules enriched within the large family of phenylpropanoid biosynthetic process. Many of 


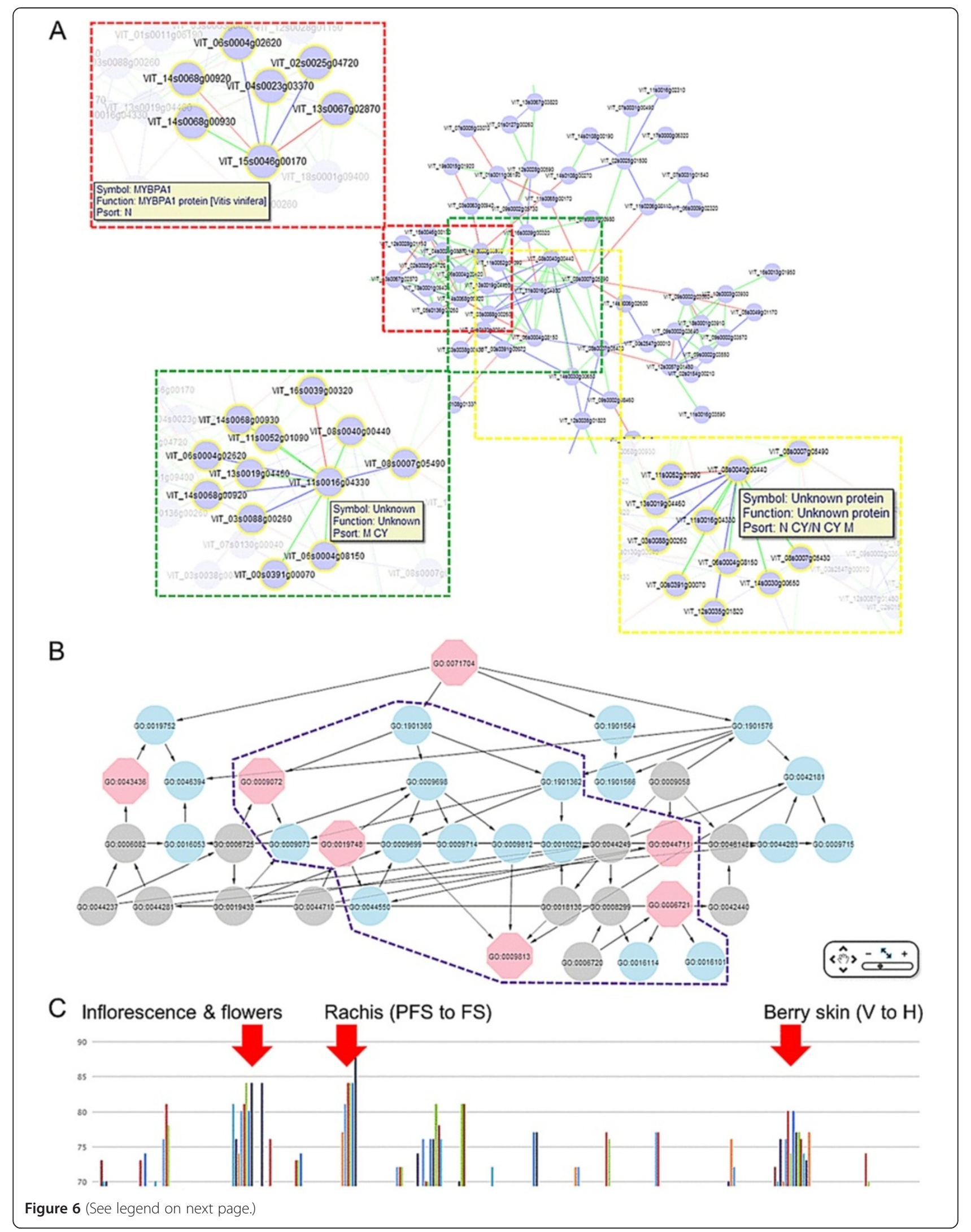


(See figure on previous page.)

Figure 6 Predicted module involved in flavonoid, amino acid and related metabolism (module All_HCCA_181). (A) Output for module 'All _HCCA_181', which contains 74 nodes and 152 edges, includes genes predominantly associated with general flavonoid, aromatic amino acid metabolic pathways and transport. Sub-network of putative grapevine MYBPA1 and two unknown genes extracted from module 'All _HCCA_181' within a neighbourhood distance of 1 are highlighted in red, green and yellow boxes, respectively. (B) Network representation of truly significant and highly enriched GO:BP terms related to flavonoid, aromatic amino acid and terpenoid metabolism are highlighted in purple boxes. Other enriched GO terms were removed from presentation for clarity. (C) Expression specificity of module 'All_HCCA_181' showing experiments in which the probesets (70\% or greater) demonstrated specific expression (Std2Gx> 1.0). PFS, post fruitset; FS, fruitset; $V$, veraison, $H$, harvest.

the genes implicated in the flavonoid pathway, including regulatory genes, have been identified here and in previous work. However, several nodes annotated as proteins of unknown function (such as VIT_11s0016g04330 and VIT_08s0040g00440), may also qualify as candidates for biosynthetic or regulatory gene products of this specialised pathway, based on their dense connectivity with genes of the flavonoid and aromatic amino acid metabolic pathway and shared expression patterns. Such genes could be potential candidates for future characterisation of phenylpropanoid metabolic and regulatory networks (Figure 6A and C).

The regulation of genes associated with photosynthesis and flavonoid metabolism displayed conserved coexpression network structures at the gene and module level across nine different plant species $[21,28]$. Both MCL and HCCA were able to partition the grapevine co-expression network efficiently and into biologically relevant modules in which genes involved in shared biological processes were successfully recovered. Thus, the co-expression analysis (both condition-independent and -dependent) performed here largely confirms previous work while revealing new putative roles for uncharacterised grapevine genes, and demonstrates the utility of the grapevine co-expression network generated in this study.

\section{Comparison to similar co-expression studies and future developments}

Currently, two other broad plant co-expression databases include grapevine microarray data [23,28]. Compared with these, VTCdb has a species-specific focus on grapevine and offers additional advantages including (1) greater transcriptome coverage for GCA, encompassing over 29,000 genes ( $>95 \%$ of the predicted genome, according to the12X v1 grape gene annotation), (2) flexibility to perform GCA based on either the $29 \mathrm{~K}$ Nimblegen array or the extensively utilised $16 \mathrm{~K}$ Affymetrix Genechip, (3) the option to choose between 'condition-independent' and 'condition-dependent' GCA, (4) the option to explore grapevine functional modules inferred from various graph clustering approaches and (5) the provision of web-based tools to enhance the functional interpretation from GCA (i.e. functional enrichment analysis, expression patterns across a wide range of experimental conditions/treatments and network visualisation). We note that despite having thorough transcriptome coverage, this study can only provide a glimpse into 'condition-specific' GCA in grapevine. Arrays of experimental conditions encompassing berry tissues and berry developmental series as well as limited stress conditions and management treatments were sufficiently represented in the public domain. A comprehensive catalogue for datasets encompassing additional stress, hormone and tissue datasets is still needed to fine-tune and facilitate the discovery of novel co-expression relationships based on condition-specific circumstances. To this end, biannual updates of the database will be conducted when new microarray experiments are published or sufficient arrays from other platforms becomes available for GCA. Nevertheless, users of VTCdb are able to perform GCA using datasets from the $16 \mathrm{~K}$ Affymetrix Genechip, which encompass a greater variety of experimental conditions (e.g. drought, salinity, heat and pathogen attack) than the Nimblegen array, albeit at the cost of transcriptome coverage. We have demonstrated that the co-expression relationships obtained using grapevine berry development, photosynthesis and flavonoid pathway-related genes were robust and could be used to identify novel transcriptional regulatory mechanisms, supported by combined network and functional analysis in plants $[49,51,54]$. These are examples of how VTCdb can be utilised to infer gene function. The predicted modules using graph clustering were of high biological relevance and may offer new biological insights into many uncharacterised genes within these modules. Due to the large proportion of uncharacterised genes within the grapevine genome, functional annotation on the basis of gene coexpression analysis and expression patterns will provide an additional tool toward gene discovery. Therefore, VTCdb offers a one-stop online platform for GCA for the grapevine research community.

\section{Conclusions}

Gene co-expression analysis of the grapevine transcriptome and the creation of an online tool to interrogate this data, provide a vital step towards uncovering additional relationships using publicly available grapevine microarray data. This meta-analysis approach has facilitated the comprehensive annotation of functions to unknown genes and the discovery of functional modules in grapevines. With the rising trend of transcriptional analyses using RNA- 
sequencing in grapevine [55-57] and on-going improvement of the methods required to process these data for GCA [58,59], the prospect for GCA using grapevine RNAsequencing data will become feasible in the future. Nevertheless, for the purposes of reverse-engineering gene coexpression networks, microarrays are currently better suited in this goal [58]. We envisage the utility and potential of VTCdb (http://vtcdb.adelaide.edu.au/home.aspx) to provide further valuable information in hypothesis-driven studies and to aid grapevine researchers in their prioritisation of gene candidates for further study towards the understanding of biological processes related to many aspects of grapevine development and metabolism.

\section{Availability and requirements}

All results discussed within this study and additional tools to query pre-constructed networks, perform additional gene co-expression, expression meta-analysis and annotation searches are available freely at VTCdb (http://vtcdb. adelaide.edu.au/home.aspx). VTCdb supports all major web-browsers, preferably Google Chrome or Mozilla Firefox for visualization and performance purposes.

\section{Additional files}

Additional file 1: Microarray datasets and associated meta-data used in the construction of the grape co-expression network. A brief description pertaining to the unique accession, title, and number of assays, reference and conditions of microarray datasets from $16 \mathrm{~K}$ Affymetrix Genechip and $29 \mathrm{~K}$ Nimblegen whole-genome array are listed in Tables S1 and S2, respectively. Figure S1 contains screenshots of the fully expanded VTCdb web interface containing various forms to perform gene co-expression analysis.

Additional file 2: A detailed description of the microarray datasets and associated meta-data used in the construction of the grape co-expression network from $16 \mathrm{~K}$ Affymetrix Genechip and $29 \mathrm{~K}$ Nimblegen whole-genome array are listed in sheets according to the respective PlexDB ID (for $16 \mathrm{~K}$ Affymetrix Genechip array) and GEO/Arrayexpress ID (for $29 \mathrm{~K}$ Nimblegen whole-genome array). Experiments that were considered potential outliers were highlighted in red.

Additional file 3: Excel file containing twelve worksheets with a list of co-expressed genes (HRR $\leq 350, P$-value $<0.01$ ) for grapevine CEB1 (Table S1), a list of all GO terms enriched associated with CEB1 co-expressed genes (Table S2), a table containing the expression specificity of CEB1 (Table S3), a list of genes and associated information from module All_MCL_6 (Table S4), a list of all GO terms enriched in module All MCL 6 (Table S5), a table containing the expression specificity of module All_MCL_6 (Table S6), a list containing the significantly connected clusters to module All MCL 6 (Table S7) screenshots of the utility and output of keyword query using 'GO:0009698' as input (Figure S1), a list of genes and associated information from module All_HCCA_181 (Table S8), and a list of all GO terms enriched in module All_HCCA_181 (Table S9), a table containing the expression specificity of module All_HCCA_181 (Table S10), a list containing the significantly connected clusters to module All_HCCA_181 (Table S11).

\section{Abbreviations}

VTCdb: Vitis Transcriptomics and co-expression database; GCA: Gene coexpression analysis; GCN: Gene co-expression network; TF: Transcription factor; GO: Gene ontology; PCC: Pearson's correlation coefficient;
SCC: Spearman's correlation coefficient; HRR: Highest reciprocal rank; MR: Mutual rank; SCS: Set count sizes; HCCA: Heuristic cluster chiselling algorithm; MCL: Markov clustering.

\section{Competing interests}

The authors declare that they have no competing interests.

\section{Authors' contributions}

DCJW conceived the study, compiled and analysed the microarray data, performed co-expression data analysis, constructed the database platform and drafted the manuscript. CMF, CS, DPD participated in co-expression data analysis, design and coordination of the study and assisted in drafting the manuscript. All authors have read and approved the final manuscript.

\section{Acknowledgements}

We gratefully acknowledge the grapevine research community for the provision of various microarray data in the public domain and the anonymous referees for providing us with constructive comments and suggestions. This work was part-supported by Australia's grape growers and winemakers through their investment body, the Grape and Wine Research and Development Corporation, with matching funds from the Australian Government (project UA 10/01). DCJW is supported by a postgraduate research scholarship from the University of Adelaide. DPD received funding from the European Union Seventh Framework Programme (FP7/2007-2013) under grant agreement 275422, which supported a Marie Curie International Outgoing Fellowship.

\section{Author details}

${ }^{1}$ School of Agriculture, Food and Wine, University of Adelaide, Adelaide 5064, South Australia, Australia. ${ }^{2}$ Department of Plant and Environmental Sciences, Section for Plant Biochemistry, University of Copenhagen, Frederiksberg 1871, Denmark.

Received: 25 March 2013 Accepted: 29 November 2013

Published: 16 December 2013

\section{References}

1. FAOSTAT. 2013 [http://faostat.fao.org]

2. Jaillon OJ, Aury JM, Noel B, Policriti A, et al: The grapevine genome sequence suggests ancestral hexaploidization in major angiosperm phyla. Nature 2007, 449(7161):463-467.

3. Velasco R, Zharkikh A, Troggio M, Cartwright DA, Cestaro A, Pruss D, Pindo M, FitzGerald LM, Vezzulli S, Reid J, et al: A high quality draft consensus sequence of the genome of a heterozygous grapevine variety. PLoS One 2007, 2(12):e1326.

4. Grimplet J, Van Hemert J, Carbonell-Bejerano P, Diaz-Riquelme J, Dickerson J, Fennell A, Pezzotti M, Martinez-Zapater J: Comparative analysis of grapevine whole-genome gene predictions, functional annotation, categorization and integration of the predicted gene sequences. BMC Research Notes 2012, 5(1):213

5. Zharkikh A, Troggio M, Pruss D, Cestaro A, Eldrdge G, Pindo M, Mitchell JT, Vezzulli S, Bhatnagar S, Fontana P, et al: Sequencing and assembly of highly heterozygous genome of vitis vinifera L. cv Pinot Noir: problems and solutions. J Biotechnol 2008, 136(1-2):38-43.

6. Forcato $C$ : Gene prediction and functional annotation in the Vitis vinifera genome. PhD Thesis 2010, 1:120.

7. Deluc L, Grimplet J, Wheatley M, Tillett R, Quilici D, Osborne C, Schooley D, Schlauch K, Cushman J, Cramer G: Transcriptomic and metabolite analyses of Cabernet Sauvignon grape berry development. BMC Genomics 2007, 8(1):429.

8. Pilati S, Perazzolli M, Malossini A, Cestaro A, Dematte L, Fontana P, Dal Ri A, Viola R, Velasco R, Moser C: Genome-wide transcriptional analysis of grapevine berry ripening reveals a set of genes similarly modulated during three seasons and the occurrence of an oxidative burst at veraison. BMC Genomics 2007, 8(1):428.

9. Grimplet J, Deluc L, Tillett R, Wheatley M, Schlauch K, Cramer G, Cushman J: Tissue-specific mRNA expression profiling in grape berry tissues. BMC Genomics 2007, 8(1):187.

10. Fasoli M, Dal Santo S, Zenoni S, Tornielli GB, Farina L, Zamboni A, Porceddu A Venturini L, Bicego M, Murino V, et al: The grapevine expression Atlas reveals a deep transcriptome shift driving the entire plant into a maturation program. Plant Cell 2012, 24(9):3489-3505. 
11. Hren M, Nikolic P, Rotter A, Blejec A, Terrier N, Ravnikar M, Dermastia M, Gruden K: Bois noir' phytoplasma induces significant reprogramming of the leaf transcriptome in the field grown grapevine. BMC Genomics 2009, 10(1):460.

12. Pastore C, Zenoni S, Tornielli GB, Allegro G, Dal Santo S, Valentini G, Intrieri C, Pezzotti M, Filippetti l: Increasing the source/sink ratio in Vitis vinifera (cv Sangiovese) induces extensive transcriptome reprogramming and modifies berry ripening. BMC Genomics 2011, 12(1):631.

13. Barrett T, Troup DB, Wilhite SE, Ledoux P, Evangelista C, Kim IF, Tomashevsky M, Marshall KA, Phillippy KH, Sherman PM, et al: NCBI GEO: archive for functional genomics data sets-10 years on. Nucleic Acids Res 2011, 39(suppl 1):D1005-D1010.

14. Parkinson H, Kapushesky M, Kolesnikov N, Rustici G, Shojatalab M, Abeygunawardena N, Berube H, Dylag M, Emam I, Farne A, et al: ArrayExpress update-from an archive of functional genomics experiments to the atlas of gene expression. Nucleic Acids Res 2009, 37(suppl 1):D868-D872.

15. Aoki K, Ogata Y, Shibata D: Approaches for Extracting Practical Information from Gene Co-expression Networks in Plant Biology. Plant Cell Physiol 2007, 48(3):381-390.

16. Usadel B, Obayashi T, Mutwil M, Giorgi FM, Bassel GW, Tanimoto M, Chow A Steinhauser D, Persson S, Provart NJ: Co-expression tools for plant biology: opportunities for hypothesis generation and caveats. Plant Cell Environ 2009, 32(12):1633-1651.

17. Ihmels J, Levy R, Barkai N: Principles of transcriptional control in the metabolic network of Saccharomyces cerevisiae. Nat Biotechnol 2004, 22(1):86-92.

18. van Noort $V$, Snel B, Huynen MA: The yeast coexpression network has a small-world, scale-free architecture and can be explained by a simple model. EMBO Rep 2004, 5(3):280-284.

19. Bergmann S, Ihmels J, Barkai N: Similarities and differences in genomewide expression data of Six organisms. PLoS Biol 2003, 2(1):e9.

20. Stuart JM, Segal E, Koller D, Kim SK: A gene-coexpression network for global discovery of conserved genetic modules. Science 2003, 302(5643):249-255.

21. Mutwil M, Klie S, Tohge T, Giorgi FM, Wilkins O, Campbell MM, Fernie AR, Usadel B, Nikoloski Z, Persson S: PlaNet: Combined Sequence and Expression Comparisons across Plant Networks Derived from Seven Species. Plant Cell 2011, 23(3):895-910.

22. Obayashi T, Hayashi S, Saeki M, Ohta H, Kinoshita K: ATTED-II provides coexpressed gene networks for Arabidopsis. Nucleic Acids Res 2009, 37(suppl 1):D987-D991.

23. Yim W, YU Y, Song K, Jang C, Lee B-M: PLANEX: the plant co-expression database. BMC Plant Biol 2013, 13(1):83.

24. Fukushima A, Nishizawa T, Hayakumo M, Hikosaka S, Saito K, Goto E, Kusano M: Exploring tomato gene functions based on coexpression modules using graph clustering and differential coexpression approaches. Plant Physiol 2012, 158(4):1487-1502.

25. Obayashi T, Nishida K, Kasahara K, Kinoshita K: ATTED-II Updates: Condition-Specific Gene Coexpression to Extend Coexpression Analyses and Applications to a Broad Range of Flowering Plants. Plant Cell Physiol 2011, 52(2):213-219.

26. Saito K, Hirai MY, Yonekura-Sakakibara K: Decoding genes with coexpression networks and metabolomics - 'majority report by precogs'. Trends Plant Sci 2008, 13(1):36-43.

27. Wang J, Li M, Deng Y, Pan Y: Recent advances in clustering methods for protein interaction networks. BMC Genomics 2010, 11(Suppl 3):S10.

28. Ogata $Y$, Suzuki H, Sakurai N, Shibata D: CoP: a database for characterizing co-expressed gene modules with biological information in plants. Bioinformatics 2010, 26(9):1267-1268.

29. Persson S, Wei H, Milne J, Page GP, Somerville CR: Identification of genes required for cellulose synthesis by regression analysis of public microarray data sets. Proc Natl Acad Sci U S A 2005, 102(24):8633-8638.

30. Hirai MY, Sugiyama K, Sawada Y, Tohge T, Obayashi T, Suzuki A, Araki R, Sakurai N, Suzuki H, Aoki K, et al: Omics-based identification of Arabidopsis Myb transcription factors regulating aliphatic glucosinolate biosynthesis. Proc Natl Acad Sci 2007, 104(15):6478-6483.

31. Dash S, Van Hemert J, Hong L, Wise RP, Dickerson JA: PLEXdb: gene expression resources for plants and plant pathogens. Nucleic Acids Res 2012, 40(D1):D1194-D1201

32. Carvalho BS, Irizarry RA: A framework for oligonucleotide microarray preprocessing. Bioinformatics 2010, 26(19):2363-2367.
33. Mutwil M, Usadel B, Schütte M, Loraine A, Ebenhöh O, Persson S: Assembly of an interactive correlation network for the arabidopsis genome using a novel Heuristic Clustering Algorithm. Plant Physiol 2010, 152(1):29-43.

34. Obayashi T, Kinoshita K: Rank of Correlation Coefficient as a Comparable Measure for Biological Significance of Gene Coexpression. DNA Research 2009, 16(5):249-260.

35. Mutwil M, Øbro J, Willats WGT, Persson S: GeneCAT—novel webtools that combine BLAST and co-expression analyses. Nucleic Acids Res 2008, 36(suppl 2):W320-W326.

36. Enright AJ, Van Dongen $\mathrm{S}$, Ouzounis CA: An efficient algorithm for large-scale detection of protein families. Nucleic Acids Res 2002, 30(7):1575-1584

37. Reimand J, Kull M, Peterson H, Hansen J, Vilo J: g:Profiler-a web-based toolset for functional profiling of gene lists from large-scale experiments. Nucleic Acids Res 2007, 35(suppl 2):W193-W200.

38. Yang X, Li J, Lee Y, Lussier YA: GO-Module: functional synthesis and improved interpretation of Gene Ontology patterns. Bioinformatics 2011, 27(10):1444-1446.

39. Grimplet J, Cramer GR, Dickerson JA, Mathiason K, Van Hemert J, Fennell AY: VitisNet: "Omics" Integration through Grapevine Molecular Networks. PLoS One 2009, 4(12):e8365.

40. Lopes CT, Franz M, Kazi F, Donaldson SL, Morris Q, Bader GD: Cytoscape Web: an interactive web-based network browser. Bioinformatics 2010, 26(18):2347-2348.

41. Coombe BG: Research on development and ripening of the grape berry Am J Enol Vitic 1992, 43(1):101-110.

42. Nicolas $P$, Lecourieux D, Gomès $E$, Delrot $S$, Lecourieux F: The grape berry-specific basic helix-loop-helix transcription factor VvCEB1 affects cell size. J Exp Bot 2013, 64(4):991-1003.

43. Ishimaru M, Smith DL, Gross KC, Kobayashi S: Expression of three expansin genes during development and maturation of Kyoho grape berries. J Plant Physiol 2007, 164(12):1675-1682.

44. Dal Santo S, Vannozzi A, Tornielli GB, Fasoli M, Venturini L, Pezzotti M, Zenoni S: Genome-wide analysis of the expansin gene superfamily reveals grapevine-specific structural and functional characteristics. PLOS One 2013, 8(4):e62206.

45. Foyer $\mathrm{CH}$, Noctor $\mathrm{G}$ : Ascorbate and glutathione: the heart of the redox hub. Plant Physiol 2011, 155(1):2-18.

46. Wei H, Persson S, Mehta T, Srinivasasainagendra V, Chen L, Page GP, Somerville C, Loraine A: Transcriptional coordination of the metabolic network in arabidopsis. Plant Physiol 2006, 142(2):762-774.

47. Nelson N, Yocum CF: Structure and function of photosystems I and II. Annu Rev Plant Biol 2006, 57(1):521-565.

48. Peter E, Grimm B: GUN4 Is required for posttranslational control of plant tetrapyrrole biosynthesis. Mol Plant 2009, 2(6):1198-1210.

49. Pan Y, Bradley G, Pyke K, Ball G, Lu C, Fray R, Marshall A, Jayasuta S, Baxter C, van Wijk R, et al: Network inference analysis identifies an APRR2-like gene linked to pigment accumulation in tomato and pepper fruits. Plant Physiol 2013, 161(3):1476-1485.

50. Horan K, Jang C, Bailey-Serres J, Mittler R, Shelton C, Harper JF, Zhu J-K Cushman JC, Gollery M, Girke T: Annotating genes of known and unknown function by large-scale coexpression analysis. Plant Physiol 2008 147(1):41-57

51. Bogs J, Jaffé FW, Takos AM, Walker AR, Robinson SP: The grapevine transcription factor VvMYBPA1 regulates proanthocyanidin synthesis during fruit development. Plant Physiol 2007, 143(3):1347-1361.

52. Terrier $\mathrm{N}$, Torregrosa L, Ageorges A, Vialet S, Verriès C, Cheynier V, Romieu C: Ectopic expression of VvMybPA2 promotes proanthocyanidin biosynthesis in grapevine and suggests additional targets in the pathway. Plant Physiol 2009, 149(2):1028-1041.

53. Hichri I, Barrieu F, Bogs J, Kappel C, Delrot S, Lauvergeat V: Recent advances in the transcriptional regulation of the flavonoid biosynthetic pathway. J Exp Bot 2011, 62(8):2465-2483.

54. Waters MT, Wang P, Korkaric M, Capper RG, Saunders NJ, Langdale JA: GLK transcription factors coordinate expression of the photosynthetic apparatus in arabidopsis. The Plant Cell Online 2009, 21(4):1109-1128.

55. Sweetman C, Wong D, Ford C, Drew D: Transcriptome analysis at four developmental stages of grape berry (Vitis vinifera cv. Shiraz) provides insights into regulated and coordinated gene expression. BMC Genomics 2012, 13(1):691.

56. Venturini L, Ferrarini A, Zenoni S, Tornielli GB, Fasoli M, Santo SD, Minio A, Buson $\mathrm{G}$, Tononi $\mathrm{P}$, Zago ED, et al: De novo transcriptome characterization 
of Vitis vinifera cv. Corvina unveils varietal diversity. BMC Genomics 2013, 14(1):1471-2164.

57. Perazzolli M, Moretto M, Fontana P, Ferrarini A, Velasco R, Moser C, Delledonne M, Pertot I: Downy mildew resistance induced by Trichoderma harzianum T39 in susceptible grapevines partially mimics transcriptional changes of resistant genotypes. BMC Genomics 2012, 13(1):1471-2164

58. Giorgi FM, Del Fabbro C, Licausi F: Comparative study of RNA-seq- and Microarray-derived coexpression networks in Arabidopsis thaliana. Bioinformatics 2013, 29(6):717-724.

59. Hong S, Chen X, Jin L, Xiong M: Canonical correlation analysis for RNA-seq co-expression networks. Nucleic Acids Res 2013, 41(8):e95.

doi:10.1186/1471-2164-14-882

Cite this article as: Wong et al:: VTCdb: a gene co-expression database for the crop species Vitis vinifera (grapevine). BMC Genomics 2013 14:882.

\section{Submit your next manuscript to BioMed Central and take full advantage of:}

- Convenient online submission

- Thorough peer review

- No space constraints or color figure charges

- Immediate publication on acceptance

- Inclusion in PubMed, CAS, Scopus and Google Scholar

- Research which is freely available for redistribution 